\title{
The Differences of Technical Elements between European National Volleyball Men and Women Teams and Their Impact in Predicting the Match Winners
}

\author{
A. Patsiaouras ${ }^{a,}$, E. Gortsila ${ }^{a}$ \\ a Department of Physical Education and Sport Science, University of Thessaly, Trikala-42100, Greece. \\ *Corresponding author Email: spats@uth.gr \\ DOI: https://doi.org/10.34256/ijpefs2137 \\ Received: 05-06-2021, Revised: 26-07-2021; Accepted: 28-07-2021; Published: 30-07-2021
}

\begin{abstract}
The purpose of this study was to identify the technical volleyball elements participating and contributing to the victory in the qualifying matches for the 2021 CEV European Men's and Women's Championship and lead to the best scoring position and qualification to the final stage. The sample consisted of the matches of the 8 teams (4 Men and 4 Women) who participated in the qualifying matches for the European Men's and Women's Championships Confédération Européenne de Volleyball (CEV) 2021. The data was analyzed using the official software of the European Confederation (CEV) (Data Volley 2 Professional). The data was then further analyzed using the SPSS 21.0 statistical package with the help of descriptive statistical analysis. Statistically significant differences were found between the teams that won and qualified for the European Championship of CEV 2021 in "reception error" and "attack error" and between the men's and women's volleyball teams in service error attack points. In addition, based on the results of this study, all coaches should focus and organize training with exercises related to these technical skills that lead to victory and qualification to improve the ability of their players to win the matches.
\end{abstract}

Keywords: Volleyball, Performance, National volleyball teams, CEV

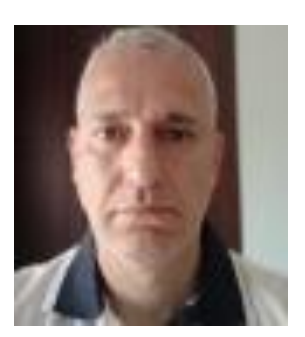

Dr. Patsiaouras Asterios, EEP is sport psychologist and volleyball coach. Currently he is working as a research fellow (permanent staff) at the Department of Physical Education and Sport Science of University of Thessaly, Greece. His area of research interests are sport psychology and sport didactic, particularly in volleyball.

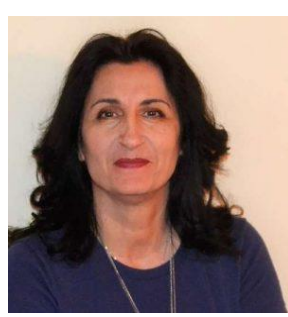

Dr. Evgenia Gortsila has a Phd on the field of Sport Science. She has worked for almost 25 years as a Physical Education teacher in Secondary Education. She has a several-year teaching experience in tertiary education, both on an undergraduate and on a postgraduate level. From 2011 to 2015, she was Headmistress at a Secondary education school. Since 2016, she has been working in the Institute of Educational Policy as a Physical Education Consultant. From 2016 until now, she has also been a member of the Inclusive Education Unit in IEP. She has practical experience in school project management and implementation as well as in the development of European projects. Her scientific interests are mainly focused on subjects related to Physical Education and Sports teaching.

\section{Introduction}

A volleyball match between two competing and equally talented teams can cause significant physical and mental stress levels to players and coaches, especially when both teams fight to their limits, thoroughly exhausting their physical potential. In such cases, especially in matches between national volleyball teams, successful teams often rely on learned patterns of actions and strategies to achieve their goal of winning the match. The evolution of coaching in volleyball, the acceptance of proposals, and their integration into the daily coaching practice of the teams is manifested in the increased homogeneity 
of the characteristics of the high-level athletes [1]. Research indicates that the top teams in volleyball show significant similarities in average player height and in-match skills' technical performance $[1,2]$. Therefore, matches between the world's top teams are usually well balanced. Understanding whether the indicators of successful execution of skills are related to the award of points in a match is useful for volleyball players and coaches in all team sports [3-7]. In any sporting activity, even in volleyball, no technical elements can be successfully performed without the player performing the skill competently. Thus, all coaches strive through proper training to develop and improve skills and isolate from the development and purification of skills and habits that lead a team to win points and win in a set or match $[8,9]$. When we observe the various technical skills in a volleyball match (of service, block, attack, reception, layout and defense), it seems logical to conclude that the team, which will make the least errors, will have the most chances to win in the match. Attacks, blocks, and services, because of the possibility that exists to achieve a point (Ace) directly, are considered scoring skills that contribute significantly to victory in a match both abroad $[4,7]$ as well as in Greece $[10,11]$. On the other hand, some other actions such as the processes of defense, the faction of the team, etc., are regarded as non-scoring skills [4], and therefore, they should, at first glance, contribute less to victory or defeat in a match.

Furthermore, the study of the relevant bibliography shows the existence of several studies in the field of volleyball. Still, due to the changes of regulations made almost every four years and the different strategies used by the coaches, there is a constant need to update the effectiveness of the technical elements, but also to demonstrate the new trends that appear in the use of skills in volleyball matches. However, it remains crucial to understand the importance and contribution of successfully executing technical elements (technical skills). In some studies $[12,13]$, it has been shown that in high-level teams, errors in technical elements may result from taking a higher level of risk than from problems arising from the application of the poor technique in the execution of the technical skill. However, there is a lack of studies in volleyball that focus on the analysis of performance factors that separate the winning from the defeated teams and contribute to their final ranking during a qualifying tournament.
Based on those above, the main objective of this research was to find out which technical elements contribute to the winning results by analyzing the differences between the first in the rating compared to the rest of the men's and women's teams in selected qualifying matches for the European Championship of CEV season 2021. It seems reasonable to do this research trying to find out whether the conclusions of previous investigations and suggestions to the coaches of high-level teams were taken into account by the coaches of the national teams and incorporated into their strategy to win a match. The purpose of this research was to investigate the influence of technical elements on the victory and defeat of teams and to examine differences between men's and women's national volleyball teams that took part in the qualifying matches for the European men's and women's 2021 Championship of Confédération Européenne de Volleyball (CEV).

\section{Materials and Methods}

\subsection{Sample}

The study sample was selected using the qualifying matches for the finals of the men's and women's European Championship (CEV) of the 2021 season. The participants of the men's and women's national teams in the matches were 8 , namely the national volleyball teams of Moldova, Cyprus, Latvia, and Spain for the men (Pool D) and the national volleyball teams of Austria, Norway, Greece, and Spain for the women (Pool C). A total of 12 matches were evaluated, and a total of 40 sets were played (19 sets for women and 21 sets for men). Women's matches were played in 19 sets where 5 matches ended with 30 sets, and one match ended with 3-1 sets. The men's matches were played in 21 sets where 3 matches ended with 3-0 sets, and 3 matches ended with 3-1 sets. There were no matches that ended in 3-2 sets for both men and women National Teams.

\subsection{Procedure}

The 12 qualifying matches for the finals of the European men's and women's championship (CEV) 2021 were first analyzed with the official data software of volleyball, and then the data was inserted into the statistical package SPSS 21.0 for further statistical processing. The teams were divided according to their ranking at the end of the championship into 2 categories high (1-2 position) and low (3-4 position). The technical elements analyzed were: a) service (total 
number of services, errors in service, passing from the opponent directly to our court after service -free ball, ace- the direct point from service), b) reception (total number of receptions, errors in reception, point for the opponent, passing the ball to the opponent-free ball), c) attack (total number of attacks, errors in attack, opponent's block in attack, point after attack), d) Block (point from block, ratio of Block success set).

\subsection{Measuring instruments}

The software approved by the European confederation of volleyball Confédération Européenne de Volleyball (CEV), Data Volley 2 Professional of the company Data Project Sr, was used to record the results of the matches of the teams that took part in the qualifying matches for the finals of the European Championship of CEV 2021 [14]. Also, all teams in Europe and worldwide use the software as mentioned above, which confirms the reliability of the information provided by this program. This software records quantitative data only and not qualitative, that is, it records, for example, the number of attacks carried out, but not the quality of the attack. The software records all the technical elements involved in a volleyball match, while in addition, errors and points gained through actions in the technical elements mentioned above are recorded.

In addition, the reliability of the observations was examined using Cohen's Kappa (intra-observer Cohen's Kappa) for 2 matches using 2 independent experts volleyball coaches with many years of experience in the matches that simultaneously recorded the matches in the sports hall and ranged between Kappa $=.94$ and Kappa $=.98$, which means that the data of the observations were reliable. If there was a discrepancy between the experts, then the video of the matches was analyzed and compared by the researchers' team.

\subsection{Statistical analysis}

Statistical analysis was carried out using SPSS v21.0. The data were analyzed using one-way ANOVA to examine any differences according to team type (National Team Men and National Team Women) and according to gender (males-females) of this sample for each factor tested.

\section{Results}

The following table 1 below shows descriptive statistics of technical elements between teams that won or lost a match.

The results of one-way ANOVA reveal statistically significant differences in technical elements between the teams that won the match and those that lost the match.

Table 2 shows the results of one-way ANOVA of technical elements between teams that won or lost a match. It was observed statistically significant differences in the total number of services, in particular, the teams that won did more services than those that lost the match $\left(p=0.013, \eta^{2}=.248\right.$, a small effect), and the points (Aces) earned with the service $\left(p=0.027, \eta^{2}=.204\right.$, a small effect). Also, the teams that lost a match made a more significant number of attempts to receive the service $\left(p=0.011, \eta^{2}=.260\right.$, a small effect) and had more errors in their reception $\left(p=0.027, \eta^{2}=.204\right.$, a small effect).

Furthermore, teams that lost the match made more mistakes in attack than teams that won a match ( $p=0.007, \eta^{2}=.288$, a small effect), while the teams that won had more excellent perceptions $(p=0.033$, $\eta^{2}=.190$, a small effect and got more points with their attack than the teams that lost a match $(p=0.019$, $\eta^{2}=.226$, a small effect.

The following table 3 shows descriptive statistics of the technical data between the teams that qualified or did not qualify for the matches for the finals of the European Championship of CEV 2021. The results of one-way ANOVA reveal statistically significant differences in technical elements between the teams that qualified for these matches and those that lost the matches and did not qualify.

Table 4 shows the results of the one-way ANOVA of technical data between the teams that qualified or did not qualify for the matches to the finals of the CEV 2021 European Championship. Statistically, significant differences were observed in the total number of service reception $\left(p=0.047, \eta^{2}=0.167\right.$, a small effect) and errors in their reception ( $p=0.027$, $\eta^{2}=0.204$, a small effect) concerning the teams that qualified. They also were statistically significantly better in excellent reception than the teams that did not qualify ( $p=0.006, \eta^{2}=0.294$, a medium effect) and less attack errors $\left(p=0.007, \eta^{2}=0.294\right.$, a small effect).

Table 5 below shows descriptive statistics of technical elements between the men's and women's 
teams that took part in the qualifying matches for the finals of the CEV 2021 European Championship.

The results of one-way ANOVA reveal statistically significant differences in technical elements between the men's and women's teams that took part in the qualifying matches for the finals of the European Championship of CEV 2021 (Table 6). There are statistically significant differences in points from the serve $\left(p=0.027, \eta^{2}=0.204\right.$, a small effect). It seems that women get more points from serving than men. Additionally, women's teams had statistically significantly more errors in reception $(p=0.027$, $\eta^{2}=0.204$, a small effect) concerning men's volleyball teams.

Table 1. Mean value and SD of technical elements between teams that won or lost a match.

\begin{tabular}{|c|c|c|c|c|}
\hline Technical element & Result & $\mathrm{N}$ & $M$ & SD \\
\hline \multirow[t]{2}{*}{ Service-total } & lost the match & 12 & 63.58 & 19.19 \\
\hline & won the match & 12 & 81.00 & 11.52 \\
\hline \multirow[t]{2}{*}{ Service-error } & lost the match & 12 & 10.25 & 3.98 \\
\hline & won the match & 12 & 10.83 & 2.25 \\
\hline \multirow[t]{2}{*}{ Servive-point } & lost the match & 12 & 3.58 & 1.73 \\
\hline & won the match & 12 & 5.50 & 2.20 \\
\hline \multirow[t]{2}{*}{ Reception-total } & lost the match & 12 & 70.17 & 10.35 \\
\hline & won the match & 12 & 53.33 & 18.23 \\
\hline \multirow[t]{2}{*}{ Reception-error } & lost the match & 12 & 5.50 & 2.20 \\
\hline & won the match & 12 & 3.58 & 1.73 \\
\hline \multirow[t]{2}{*}{ Reception-positive } & lost the match & 12 & 45.92 & 8.49 \\
\hline & won the match & 12 & 48.25 & 4.39 \\
\hline \multirow[t]{2}{*}{ Reception-excellent } & lost the match & 12 & 25.25 & 7.10 \\
\hline & won the match & 12 & 30.67 & 4.25 \\
\hline \multirow[t]{2}{*}{ Attack-total } & lost the match & 12 & 100.00 & 26.444 \\
\hline & won the match & 12 & 96.42 & 35.321 \\
\hline \multirow[t]{2}{*}{ Attack-error } & lost the match & 12 & 9.75 & 2.10 \\
\hline & won the match & 12 & 6.08 & 3.70 \\
\hline
\end{tabular}


Attack-block

Attack-points

Block

lost the match

won the match

lost the match

won the match

lost the match

won the match

12

12

12

12

12

12
8.00

2.63

6.17

3.54

12.99

10.40

Table 2. One - way ANOVA of technical elements between teams that won or lost a match.

\begin{tabular}{|c|c|c|c|c|c|c|c|}
\hline Technical Elements & Groups & SS & Df & MS & $\mathrm{F}$ & $\mathrm{p}$ & $\eta^{2}$ \\
\hline Service-total & $\begin{array}{l}\text { Between groups } \\
\text { Within groups } \\
\text { Total }\end{array}$ & $\begin{array}{l}1820.042 \\
5510.917 \\
7330.958\end{array}$ & $\begin{array}{l}1 \\
22 \\
23\end{array}$ & $\begin{array}{l}1820.042 \\
250.496\end{array}$ & 7,27 & $0.013^{* *}$ & 0.248 \\
\hline Service-error & $\begin{array}{l}\text { Between groups } \\
\text { Within groups } \\
\text { Total }\end{array}$ & $\begin{array}{l}2.042 \\
229.917 \\
231.958\end{array}$ & $\begin{array}{l}1 \\
22 \\
23\end{array}$ & $\begin{array}{l}2.042 \\
10.451\end{array}$ & 0.20 & 0.663 & 0.009 \\
\hline Service-point & $\begin{array}{l}\text { Between groups } \\
\text { Within groups } \\
\text { Total }\end{array}$ & $\begin{array}{l}22.042 \\
85.917 \\
107.958\end{array}$ & $\begin{array}{l}1 \\
22 \\
23\end{array}$ & $\begin{array}{l}22.042 \\
3.905\end{array}$ & 5.64 & $0.027 *$ & 0.204 \\
\hline Reception-total & $\begin{array}{l}\text { Between groups } \\
\text { Within groups } \\
\text { Total }\end{array}$ & $\begin{array}{l}1700.167 \\
4834.333 \\
6534.500\end{array}$ & $\begin{array}{l}1 \\
22 \\
23\end{array}$ & $\begin{array}{l}1700.167 \\
219.742\end{array}$ & 7.737 & $0.011 * *$ & 0.260 \\
\hline Reception-error & $\begin{array}{l}\text { Between groups } \\
\text { Within groups } \\
\text { Total }\end{array}$ & $\begin{array}{l}22.042 \\
85.917 \\
107.958\end{array}$ & $\begin{array}{l}1 \\
22 \\
23\end{array}$ & $\begin{array}{l}22.042 \\
3.905\end{array}$ & 5.644 & $0.027 *$ & 0.204 \\
\hline Reception-positive & $\begin{array}{l}\text { Between groups } \\
\text { Within groups } \\
\text { Total }\end{array}$ & $\begin{array}{l}32.667 \\
1005.167 \\
1037.833\end{array}$ & $\begin{array}{l}1 \\
22 \\
23\end{array}$ & $\begin{array}{l}32.667 \\
45.689\end{array}$ & 0.715 & 0.407 & 0.031 \\
\hline Reception-excellent & $\begin{array}{l}\text { Between groups } \\
\text { Within groups } \\
\text { Total }\end{array}$ & $\begin{array}{l}176.042 \\
752.917 \\
928.958\end{array}$ & $\begin{array}{l}1 \\
22 \\
23\end{array}$ & $\begin{array}{l}176.042 \\
34.223\end{array}$ & 5.144 & $0.033 *$ & 0.190 \\
\hline Attack-total & $\begin{array}{l}\text { Between groups } \\
\text { Within groups } \\
\text { Total }\end{array}$ & $\begin{array}{l}77.042 \\
21414.917 \\
21491.958\end{array}$ & $\begin{array}{l}1 \\
22 \\
23\end{array}$ & $\begin{array}{l}77.042 \\
973.405\end{array}$ & 0.079 & 0.781 & 0.004 \\
\hline
\end{tabular}




\begin{tabular}{|c|c|c|c|c|c|c|c|}
\hline Attack-error & $\begin{array}{l}\text { Between groups } \\
\text { Within groups } \\
\text { Total }\end{array}$ & $\begin{array}{l}80.667 \\
199.167 \\
279.833\end{array}$ & $\begin{array}{l}1 \\
22 \\
23\end{array}$ & $\begin{array}{l}80.667 \\
9.053\end{array}$ & 8.910 & $0.007 * *$ & 0.288 \\
\hline Attack-block & $\begin{array}{l}\text { Between groups } \\
\text { Within groups } \\
\text { Total }\end{array}$ & $\begin{array}{l}20.167 \\
213.667 \\
233.833\end{array}$ & $\begin{array}{l}1 \\
22 \\
23\end{array}$ & $\begin{array}{l}20.167 \\
9.712\end{array}$ & 2.076 & 0.164 & 0.086 \\
\hline Attack-points & $\begin{array}{l}\text { Between groups } \\
\text { Within groups } \\
\text { Total }\end{array}$ & $\begin{array}{l}888,167 \\
3043.167 \\
3931.333\end{array}$ & $\begin{array}{l}1 \\
22 \\
23\end{array}$ & $\begin{array}{l}888.167 \\
138.326\end{array}$ & 6.421 & $0.019 *$ & 0.226 \\
\hline Block & $\begin{array}{l}\text { Between groups } \\
\text { Within groups } \\
\text { Total }\end{array}$ & $\begin{array}{l}20.167 \\
213.667 \\
233.833\end{array}$ & $\begin{array}{l}1 \\
22 \\
23\end{array}$ & $\begin{array}{l}20.167 \\
9.712\end{array}$ & 2.076 & 0.164 & 0.086 \\
\hline
\end{tabular}

*Significant at 0.050 level

**Significant at 0.01 level

Table 3. Mean value and SD of technical elements between teams that qualified or did not qualify for the finals of the CEV 2021 European Championship.

\begin{tabular}{|c|c|c|c|c|}
\hline Technical element & Result & $\mathrm{N}$ & $M$ & SD \\
\hline \multirow[t]{2}{*}{ Service-total } & not qualified & 12 & 68.50 & 21.33 \\
\hline & qualified & 12 & 76.08 & 13.42 \\
\hline \multirow[t]{2}{*}{ Service-error } & not qualified & 12 & 10.67 & 3.89 \\
\hline & qualified & 12 & 10.42 & 2.43 \\
\hline \multirow[t]{2}{*}{ Servive-point } & not qualified & 12 & 3.92 & 1.62 \\
\hline & qualified & 12 & 5.17 & 2.52 \\
\hline \multirow[t]{2}{*}{ Reception-total } & not qualified & 12 & 68.50 & 13.04 \\
\hline & qualified & 12 & 55.00 & 18.02 \\
\hline \multirow[t]{2}{*}{ Reception-error } & not qualified & 12 & 5.50 & 1.93 \\
\hline & qualified & 12 & 3.58 & 2.02 \\
\hline \multirow[t]{2}{*}{ Reception-positive } & not qualified & 12 & 45.33 & 8.29 \\
\hline & qualified & 12 & 48.83 & 4.35 \\
\hline
\end{tabular}




\section{Reception-excellent}

Attack-total

Attack-error

Attack-block

Attack-points

Block not qualified

qualified

not qualified

qualified

not qualified

qualified

not qualified

qualified

not qualified

qualified

not qualified

qualified

12

12

12

12

12

12

12

12

12

12

12

12
24.58

5.92

$31.33 \quad 4.96$

$99.17 \quad 26.64$

$97.25 \quad 35.25$

$9.75 \quad 2.09$

$\begin{array}{ll}6.08 & 3.70\end{array}$

$\begin{array}{ll}7.58 & 2.54\end{array}$

$\begin{array}{ll}6.58 & 3.78\end{array}$

$36.17 \quad 14.60$

$43.50 \quad 10.72$

$7.00 \quad 3.22$

$7.17 \quad 3.30$

Table 4. One - way ANOVA of technical elements between teams that qualified or did not qualify for the matches.

\begin{tabular}{|c|c|c|c|c|c|c|c|}
\hline Technical Elements & Groups & SS & Df & MS & $\mathrm{F}$ & $\mathrm{p}$ & $\eta^{2}$ \\
\hline Service-total & $\begin{array}{l}\text { Between groups } \\
\text { Within groups } \\
\text { Total }\end{array}$ & $\begin{array}{l}345.042 \\
6985.917 \\
7330.958\end{array}$ & $\begin{array}{l}1 \\
22 \\
23\end{array}$ & $\begin{array}{l}345.042 \\
317.542\end{array}$ & 1.087 & 0.309 & 0.047 \\
\hline Service-error & $\begin{array}{l}\text { Between groups } \\
\text { Within groups } \\
\text { Total }\end{array}$ & $\begin{array}{l}0.375 \\
231.583 \\
231.958\end{array}$ & $\begin{array}{l}1 \\
22 \\
23\end{array}$ & $\begin{array}{l}.375 \\
10.527\end{array}$ & 0.036 & 0.852 & 0.002 \\
\hline Servive-point & $\begin{array}{l}\text { Between groups } \\
\text { Within groups } \\
\text { Total }\end{array}$ & $\begin{array}{l}9.375 \\
98.583 \\
107.958\end{array}$ & $\begin{array}{l}1 \\
22 \\
23\end{array}$ & $\begin{array}{l}9.375 \\
4.481\end{array}$ & 2.092 & 0.162 & 0.087 \\
\hline Reception-total & $\begin{array}{l}\text { Between groups } \\
\text { Within groups } \\
\text { Total }\end{array}$ & $\begin{array}{l}1093.500 \\
5441.000 \\
6534.500\end{array}$ & $\begin{array}{l}1 \\
22 \\
23\end{array}$ & $\begin{array}{l}1093.500 \\
247.318\end{array}$ & 4.421 & $0.047 *$ & 0.167 \\
\hline Reception-error & $\begin{array}{l}\text { Between groups } \\
\text { Within groups } \\
\text { Total }\end{array}$ & $\begin{array}{l}22.042 \\
85.917 \\
107.958\end{array}$ & $\begin{array}{l}1 \\
22 \\
23\end{array}$ & $\begin{array}{l}22.042 \\
3.905\end{array}$ & 5.644 & $0.027 *$ & 0.204 \\
\hline
\end{tabular}




\begin{tabular}{|c|c|c|c|c|c|c|c|}
\hline Reception-positive & $\begin{array}{l}\text { Between groups } \\
\text { Within groups } \\
\text { Total }\end{array}$ & $\begin{array}{l}73.500 \\
964.333 \\
1037.833\end{array}$ & $\begin{array}{l}1 \\
22 \\
23\end{array}$ & $\begin{array}{l}73.500 \\
43.833\end{array}$ & 1.677 & 0.209 & 0.071 \\
\hline Reception-excellent & $\begin{array}{l}\text { Between groups } \\
\text { Within groups } \\
\text { Total }\end{array}$ & $\begin{array}{l}273.375 \\
655.583 \\
928.958\end{array}$ & $\begin{array}{l}1 \\
22 \\
23\end{array}$ & $\begin{array}{l}273.375 \\
29.799\end{array}$ & 9.174 & $0.006 * *$ & 0.294 \\
\hline Attack-total & $\begin{array}{l}\text { Between groups } \\
\text { Within groups } \\
\text { Total }\end{array}$ & $\begin{array}{l}22.042 \\
21469.917 \\
21491.958\end{array}$ & $\begin{array}{l}1 \\
22 \\
23\end{array}$ & $\begin{array}{l}22.042 \\
975.905\end{array}$ & 0.023 & 0.882 & 0.001 \\
\hline Attack-error & $\begin{array}{l}\text { Between groups } \\
\text { Within groups } \\
\text { Total }\end{array}$ & $\begin{array}{l}80.667 \\
199.167 \\
279.833\end{array}$ & $\begin{array}{l}1 \\
22 \\
23\end{array}$ & $\begin{array}{l}80.667 \\
9.053\end{array}$ & 8.910 & $0.007 * *$ & 0.288 \\
\hline Attack-block & $\begin{array}{l}\text { Between groups } \\
\text { Within groups } \\
\text { Total }\end{array}$ & $\begin{array}{l}6.000 \\
227.833 \\
233.833\end{array}$ & $\begin{array}{l}1 \\
22 \\
23\end{array}$ & $\begin{array}{l}6.000 \\
10.356\end{array}$ & 0.579 & 0.455 & 0.026 \\
\hline Attack-points & $\begin{array}{l}\text { Between groups } \\
\text { Within groups } \\
\text { Total }\end{array}$ & $\begin{array}{l}322.667 \\
3608.667 \\
3931.333\end{array}$ & $\begin{array}{l}1 \\
22 \\
23\end{array}$ & $\begin{array}{l}322.667 \\
164.030\end{array}$ & 1.967 & 0.175 & 0.082 \\
\hline Block & $\begin{array}{l}\text { Between groups } \\
\text { Within groups } \\
\text { Total }\end{array}$ & $\begin{array}{l}0.167 \\
233.667 \\
233.833\end{array}$ & $\begin{array}{l}1 \\
22 \\
23\end{array}$ & $\begin{array}{l}.167 \\
10.621\end{array}$ & 0.016 & 0.901 & 0.001 \\
\hline
\end{tabular}

*Significant at 0.050 level

**Significant at 0.001 level

Table 5. Mean and SD of technical elements between the men's and women's teams in the matches for CEV 2021 European Championship.

\begin{tabular}{lllll}
\hline Technical element & Team & N & M & SD \\
\hline Service-total & males & 12 & 77.33 & 16.37 \\
& females & 12 & 67.25 & 18.52 \\
Service-error & males & 12 & & \\
& females & 12 & 11.50 & 3.21 \\
Servive-point & males & 12 & 9.58 & 2.97 \\
& females & 12 & 3.58 & 1.83 \\
& & & 5.50 & 2.11
\end{tabular}


Reception-total

Reception-error

Reception-positive

Reception-excellent

Attack-total

Attack-error

Attack-block

Attack-points

Block males

females

males

females

males

females

males

females

males

females

males

females

males

females

males

females

males

females

12

12

12

12

12

12

12

12

12

12

12

12

12

12

12

12

12

12
65.83

14.94

$57.67 \quad 18.29$

3.58

$5.50 \quad 2.11$

$49.17 \quad 4.78$

$45.00 \quad 7.87$

$26.17 \quad 5.78$

$29.75 \quad 6.64$

$95.42 \quad 16.94$

$101.00 \quad 40.62$

$\begin{array}{ll}7.50 & 3.18\end{array}$

$8.33 \quad 3.87$

$\begin{array}{ll}7.50 & 2.54\end{array}$

$6.67 \quad 3.80$

$44.42 \quad 12.92$

$35.25 \quad 12.02$

$7.50 \quad 2.54$

$\begin{array}{ll}6.67 & 3.80\end{array}$

Table 6. One - way ANOVA of technical elements between men's and women's volleyball teams.

\begin{tabular}{|l|l|l|l|l|l|l|l|}
\hline Technical Elements & Groups & SS & Df & MS & F & p & $\eta^{2}$ \\
\hline Service-total & Between groups & 610.042 & 1 & 610.042 & & & \\
& Within groups & 6720.917 & 22 & 305.496 & 1.997 & 0.172 & 0.083 \\
& Total & 7330.958 & 23 & & & & \\
\hline Service-error & Between groups & 22.042 & 1 & 22.042 & \multirow{2}{*}{2.310} & 0.143 & 0.095 \\
& Within groups & 209.917 & 22 & 9.542 & & & \\
\hline
\end{tabular}




\begin{tabular}{|c|c|c|c|c|c|c|c|}
\hline Servive-point & $\begin{array}{l}\text { Between groups } \\
\text { Within groups } \\
\text { Total }\end{array}$ & $\begin{array}{l}22.042 \\
85.917 \\
107.958\end{array}$ & $\begin{array}{l}1 \\
22 \\
23\end{array}$ & $\begin{array}{l}22.042 \\
3.905\end{array}$ & 5.644 & $0.027 *$ & 0.204 \\
\hline Reception-total & $\begin{array}{l}\text { Between groups } \\
\text { Within groups } \\
\text { Total }\end{array}$ & $\begin{array}{l}400.167 \\
6134.333 \\
6534.500\end{array}$ & $\begin{array}{l}1 \\
22 \\
23\end{array}$ & $\begin{array}{l}400.167 \\
278.833\end{array}$ & 1.435 & 0.244 & 0.061 \\
\hline Reception-error & $\begin{array}{l}\text { Between groups } \\
\text { Within groups } \\
\text { Total }\end{array}$ & $\begin{array}{l}22.042 \\
85.917 \\
107.958\end{array}$ & $\begin{array}{l}1 \\
22 \\
23\end{array}$ & $\begin{array}{l}22.042 \\
3.905\end{array}$ & 5.644 & $0.027 *$ & 0.204 \\
\hline Reception-positive & $\begin{array}{l}\text { Between groups } \\
\text { Within groups } \\
\text { Total }\end{array}$ & $\begin{array}{l}104.167 \\
933.667 \\
1037.833\end{array}$ & $\begin{array}{l}1 \\
22 \\
23\end{array}$ & $\begin{array}{l}104.167 \\
42.439\end{array}$ & 2.454 & 0.131 & 0.100 \\
\hline Reception-excellent & $\begin{array}{l}\text { Between groups } \\
\text { Within groups } \\
\text { Total }\end{array}$ & $\begin{array}{l}77.042 \\
851.917 \\
928.958\end{array}$ & $\begin{array}{l}1 \\
22 \\
23\end{array}$ & $\begin{array}{l}77.042 \\
38.723\end{array}$ & 1.990 & 0.172 & 0.083 \\
\hline Attack-total & $\begin{array}{l}\text { Between groups } \\
\text { Within groups } \\
\text { Total }\end{array}$ & $\begin{array}{l}187.042 \\
21304.917 \\
21491.958\end{array}$ & $\begin{array}{l}1 \\
22 \\
23\end{array}$ & $\begin{array}{l}187.042 \\
968.405\end{array}$ & 0.193 & 0.665 & 0.009 \\
\hline Attack-error & $\begin{array}{l}\text { Between groups } \\
\text { Within groups } \\
\text { Total }\end{array}$ & $\begin{array}{l}4.167 \\
275.667 \\
279.833\end{array}$ & $\begin{array}{l}1 \\
22 \\
23\end{array}$ & $\begin{array}{l}4.167 \\
12.530\end{array}$ & 0.333 & 0.570 & 0.015 \\
\hline Attack-block & $\begin{array}{l}\text { Between groups } \\
\text { Within groups } \\
\text { Total }\end{array}$ & $\begin{array}{l}4.167 \\
229.667 \\
233.833\end{array}$ & $\begin{array}{l}1 \\
22 \\
23\end{array}$ & $\begin{array}{l}4.167 \\
10.439\end{array}$ & 0.399 & 0.534 & 0.018 \\
\hline Attack-points & $\begin{array}{l}\text { Between groups } \\
\text { Within groups } \\
\text { Total }\end{array}$ & $\begin{array}{l}504.167 \\
3427.167 \\
3931.333\end{array}$ & $\begin{array}{l}1 \\
22 \\
23\end{array}$ & $\begin{array}{l}504.167 \\
155.780\end{array}$ & 3.236 & 0.086 & 0.128 \\
\hline Block & $\begin{array}{l}\text { Between groups } \\
\text { Within groups } \\
\text { Total }\end{array}$ & $\begin{array}{l}4.167 \\
229.667 \\
233.833\end{array}$ & $\begin{array}{l}1 \\
22 \\
23\end{array}$ & $\begin{array}{l}4.167 \\
10.439\end{array}$ & .399 & 0.534 & 0.018 \\
\hline
\end{tabular}

*Significant at 0.050 level

***Significant at 0.001 level

\section{Discussion}

From the study results, it seems that the points earned by service are a reliable indicator for winning a match and ranking the team in a good scoring position in qualifying matches. The results of the statistical analysis that the points earned by serving are an excellent and stable indicator of the effectiveness of a team are in line with other studies, since Charitonidis, Patsiaouras, and Charitonidis [10] 
found that it is a predictor of winning or losing European national teams but also in the Professional League of men [11]. More specifically, Patsiaouras et al. [7], in their study, found that "services point" are decisive factors for winning or losing a match, while noting that avoiding mistakes (errors) during the attack could significantly contribute to winning a match.

Furthermore, the results of the study are consistent with the research of Zetou, Tsigilis, Moustakidis, and Komninakidou [15], where the service and especially the service Aces that are achieved directly with the service, is an essential factor that can be used in predicting the victory or defeat of a team in a match. However, other research supports the fact that the attack is also a determining factor of victory or loss in a volleyball match, especially at a very high level $[9,16]$. The results of this study highlight the importance of the point from service to victory. In agreement with the investigation of Marelic et al. [17], the team that had a better service tends to win more sets. Errors in the serve were also less common in the winning teams than in the other teams, but also in the men's teams than in the women's volleyball teams in fact, many researchers believe that teams that are at a disadvantage in the set tend to risk more on the serve, probably because they have nothing to lose $[17,18]$. By risking more on serve, these teams fail more often, increasing the percentage of mistakes made [17]. On the other hand, if the service is risky, the opponent's reception will be more difficult, multiplying the probability of the mistake, which we see happening mainly in women's teams. The study showed that teams that won a match and/or qualified were better in service perception, having less errors in service perception compared to teams that lost the match and/or did not qualified. It is essential, therefore, not only to improve the effectiveness of the service perception but the service too, since it is considered a final action and as an offensive action that starts the match can end up with an immediate point $[17,19]$. The attack is another important technical element in volleyball, providing statistical data on how well a team is making successful kill attempts, measuring precisely by the hitting percentage. This information is very important and useful for coaches and players in determining how well a team capitalizes on kill opportunities. Study results showed that teams lost the match due to more mistakes in attack than teams that won a match participating in the European Championship of CEV 2021. Furthermore, teams that won a match got more points with their attack than the teams that lost the match. Results are in line with previous studies that pointed out the importance of the attack for winning a match [4, 7, 9-11]. Attack errors occurred when a hitter attempts to send the ball across the net and to the opponent team's side but fails to do so, or if they do, it is done illegally according to the volleyball rules of the game. These errors are monitored and kept track of in player and team statistics. The study revealed that teams that qualified made fewer attack errors than teams that were not qualified in agreement with previous studies $[11,16$, 19].

Concerning the other technical elements examined in the study, it emerged that were not contributed statistically significantly to the effort to distinguish a team and achieve a better position and qualification in volleyball tournaments. This does not mean, however, that coaches should not give proper importance to these technical elements, since other studies analyzed teams that participated in Olympic matches showed that the attack and especially the reduced mistakes in the attack and the avoidance of bad reception are determining factors for winning or losing in a match [9- 11].

\section{Conclusions}

The main conclusion of this study is that the attack is an important and decisive factor for the performance of volleyball teams since, in this way, more points are won in a match. This study confirms that effective service and attack are variables that can be used to predict success. Therefore, service training, reception, and attack are crucial factors and should be taken into account at different points and moments during the match, using different strategies and scenarios that can cause imbalances between teams at the same level in volleyball. Observing the differences between the two genders (men volleyball teams vs woman volleyball teams), women are more effective and get more points from serving than men. They tend to risk a lot in service to win an ace, therefore they have statistically significantly more errors in reception about men's volleyball teams. The strong point of this study was that all the significant technical elements that could lead to win or lose a volleyball match were taken into account. The weak point of this study was that the statistically significant results that have been observed revealing to have a small effect, perhaps due to the small available data of the research. Further future research, including data from more teams and matches, should explore the contribution of factors such as the defense system, the initial line-up, the 
placement of players on the field in winning or losing a match and provide a guide giving helpful information for coaches what factors other than technical elements should pay attention to the critical points of a set or match to earn the points and qualify for CEV ranking matches.

\section{References}

[1] J. Sheppard, T.J. Gabbett, L. Stanganelli, An Analysis of Playing Positions in Elite Men's Volleyball: Considerations for Competition Demands and Physiologic Characteristics, Journal of Strength and Conditioning Research, 23 (2009) 1858-1866. [DOI] [PubMed]

[2] T. Gabbett, G. Boris, D. Nathan, The use of physiological, anthropometric, and skill data to predict selection in a talent-identified junior volleyball squad, Journal of Sport Science, 25 (2007) 1337-1344. [DOI] [PubMed]

[3] R. Lobietti, S. Coleman, E. Pizzichillo, F. Merni, Landing techniques in volleyball, Journal of Sport Science, 28 (2010) 1469-1476. [DOI] [PubMed]

[4] R. Marcelino, I. Mesquita, J. Sampaio, J. Moraes, Study of performance indicators in male volleyball according to the set results, Brazilian Journal of Physical Education and Sport, 24 (2010) 69-78. [DOI]

[5] M. Miskin, G. Fellingham, L. Florence, Skill Importance in Women's Volleyball, Journal of Quantitative Analysis in Sports, 6 (2010) 1-14. [DOI]

[6] J. Palao, J. Santos, A. Ureña, Effect of setter's position on the spike in volleyball, Journal of Human Movement Studies, 48 (2005) 25-40.

[7] A. Patsiaouras, K. Charitonidis, A. Moustakidis,

D. Kokaridas, Comparison of technical skills effectiveness of men's National Volleyball teams, International Journal of Performance Analysis in Sport, 9 (2009) 1-7. [DOI]

[8] T. Karalić, N. Marelić, A. Vujmilović, Structure of isolated precision factors of the male volleyball players, Sport Logia, 8 (2012) 69-77. [DOI]

[9] A. Patsiaouras, A. Moustakidis, K. Charitonidis, D. Kokaridas, Volleyball technical skills as winning and qualification factors during Olympic Matches 2008, International Journal of
Performance Analysis in Sport, 10 (2010) 115120. [DOI]

[10] K. Charitonidis, A. Patsiaouras, M. Charitonidi, Comparison of the effectiveness of the technical elements of the Greek national volleyball team with other European national teams, Inquiries in Sport \& Physical Education, 5 (2007) 431-436.

[11] M. Charitonidi, A. Patsiaouras, K. Charitonidis, M.P. Kyriazides, The effectiveness of technical elements in the Greek professional league of men in volleyball, Inquiries in Sport \& Physical Education, 16 (2018) 1-7.

[12] N. Bergeles, K. Barzouka, M. Nikolaidou, Performance of male and female setters and attackers on Olympic-level Volleyball team, International Journal of Performance Analysis in Sport, 9 (2009) 141-148. [DOI]

[13] B. Buscà, J. Febrer, Temporal fight between the middle blocker and the setter in high level volleyball, Revista Internacional de Medicina y Ciencias de la Actividad Fisica y del Deporte, 12 (2012) 313-327.

[14] Data Project (2005). Data Volley 2 Professional. Retrieved June 14, 2006, from http://www.dataproject.com/download/downlo ad file.aspx?CP=DVW2\&filename=eDV2 Setup Lite.exe\&tipo $=4 \&$ orig $=\% 2$ fdownload $\% 2$ fdow nload programmi.aspx $\% 3$ ftipo $\% 3 \mathrm{~d} 4 \% 26 \mathrm{CP} \% 3$ dDVW2

[15] E. Zetou, N. Tsigilis, A. Moustakidis, A. Komninakidou, Playing characteristics of men's Olympic Volleyball teams in complex II, International Journal of Performance Analysis in Sport, 6 (2006) 172-177. [DOI]

[16] A. Patsiaouras, A. Moustakidis, K. Charitonidis, D. Kokaridas, Technical Skills Leading in Winning or Losing Volleyball Matches During Beijing Olympic Matchs, Journal of Physical Education and Sport, 11 (2011) 39-42.

[17] N. Marelic, T. Resetar, V. Jankovic, Discriminant analysis of the sets won and the sets lost by one team in a1 Italian volleyball league - a case study, Kinesiology, 36 (2004) 75-82.

[18] P. João, N. Leite, I. Mesquita, J. Sampaio, Sex differences in discriminative power of volleyball match related statistics, Perceptual and Motor 
Skills, 111 (2010) 893-900. [DOI]

[19] A. Patsiaouras, D. Kokaridas Technical skills predictive of winning at CEV Volleyball Men"s Champions League: Identification and importance, Trends in Sport Sciences, 2(26) (2019) 71-76. [DOI]

\section{Funding}

No funding was received for conducting this study.

\section{Authors Contribution}

Both the authors equally contributed to this work. Also, both of them read and approved the final version of the manuscript.

\section{Conflict of interest}

The Authors have no conflicts of interest to declare that they are relevant to the content of this article.

\section{Does this article screened for similarity?}

Yes

\section{About The License}

(C) The Author(s) 2021. The text of this article is open access and licensed under a Creative Commons Attribution 4.0 International License 